\title{
Analysis of Medicinally Important Phytocompounds from Adina cordifolia Leaves
}

\author{
Pratima Raypa $^{1^{*}}$, A.K. Verma ${ }^{1}$, Salil Tewari ${ }^{2}$ and Ashutosh Dubey ${ }^{1^{*}}$ \\ ${ }^{1}$ Department of Biochemistry, ${ }^{2}$ Department of Genetics and Plant breeding, College of \\ Agriculture, G. B. Pant Univ. of Ag. \& Tech., Pantnagar-263145, \\ U. S. Nagar, Uttarakhand, India \\ *Corresponding author
}

\begin{tabular}{|l|}
\hline Ke y w or d s \\
$\begin{array}{l}\text { Adina cordifolia, } \text { Phenol, } \\
\text { Flavonoid, trans- } \\
\text { squalene, GC-MS }\end{array}$ \\
\hline Article Info \\
\hline $\begin{array}{l}\text { Accepted: } \\
22 \text { October } 2018 \\
\text { Available Online: } \\
10 \text { November } 2018\end{array}$ \\
\hline
\end{tabular}

A B S T R A C T

The present study was aimed to evaluate the bioactive phytochemical constituents of Adina cordifolia leaves, with this broad objectives the total phenolic contents (TPC), total flavonoid contents (TFC), total antioxidant potential and GC-MS analysis of leaf extracts were carried out. For the identification of medicinally important compounds in Adina cordifolia leaf, extracts were prepared using cold extraction method in different solvents of varying polarities viz., chloroform, ethyl acetate, acetone and methanol. Total phenolic content of plant extracts was analysed using Folin-ciocalteu reagent, total flavonoid content was estimated by using aluminium chloride and total antioxidant activity was estimated by formation of a green phosphate $\mathrm{Mo}(\mathrm{V})$ complex at acid $\mathrm{pH}$. Gas Chromatography Mass Spectroscopy was performed to identify phytocompounds present in plant extracts using National Institute of Standards and Technology (NIST) library. The highest total phenolic content and flavonoid were found in acetone extract of Adina cordifolia leaf. Total antioxidant activity was highest in methanol extract. A wide range of fatty acids and phytocompounds were also identified having antibacterial, antifungal and anti-inflammatory activities. The study concludes that Adina cordifolia have many biologically important compounds, so it can be recommended as a plant of pharmaceutical importance.

\section{Introduction}

Several plants contain a variety of phytopharmaceuticals with vital applications among the fields of agriculture and medical speciality. Plants have great potential uses as drugs and pharmacopoeil medication as a large proportion of the world population depends on traditional medicines of plant origin due to the inadequate supply and high prices of conventional modern medicine. These medicinal plants have provided the numerous plants derived therapeutic agents which play a very important role for the development of novel drug leads for the treatment and hindrance of diseases.

Haldu (Adina cordifolia), deciduous tree of subfamily Cinchonoideae, family Rubiaceae, is native of which is found Southern Asia, 
from India and Srilanka east to southern China and Vietnam. It is found scattered in deciduous forests throughout the greater part of India, ascending to an altitude of $900 \mathrm{~m}$ in sub-Himalayan tract. A. cordifolia is included in threatened species (www.fes.org.in).

A. cordifolia has been used in oriental medicine since ancient times as an essential component of various antiseptic and febrifuge prescriptions (Chopra et al., 1956). The bark is acrid, bitter pungent, tonic, vulnerary and aphrodisiac and is used in biliousness. The roots are used as an astringent in dysentery (Chadha, 1985).

A. cordifolia had been also evaluated for its anti-ulcer potential active constituent showed interesting $\mathrm{H}^{+} / \mathrm{K}^{+}$ATPase inhibitory activity (Kasinadhuni et al., 1999). Four compounds isolated from the stem of Adina cordifolia were identified as stigmasta-5, 22-diene-3P-Oa-rhamnopyranosyl-(1-4)-P-Dxylopyranoside, a-amyrin, octacosanol and naringenin-7methyl ether-4'-O-a-rhamnopyranoside on the basis of spectral and chemical evidence (Rokade and Pawar 2013).

In vitro propagation through apical buds is the best possible means for in situ conservation of Adina cordifolia, a threatened species, to produce a large numbers of plants in a short span of time. Adina cordifolia was very well established in vitro conditions in presence of MS medium supplemented $2 \mathrm{mg} / \mathrm{L}$ BAP or $0.5 \mathrm{mg} / \mathrm{L}$ NAA alone (Raypa et al., 2013).

The major compounds identified in the extracts of Mitragyna parvifolia leaf (Rubiaceae) were butanoic acid, 2-ethylhexyl ester (19.36\%), 4 methyl mannose (53.13\%), mitraphylline $(21.59 \%)$ and isomitraphylline (3.37\%). Among these, compound mitraphylline is known for its antiinflammatory, antiproliferative activities (Vasmatkar et al., 2014).

\section{Materials and Methods}

\section{Materials}

The leaves of Adina cordifolia were collected from Agro-forestry Research Centre (AFRC) Haldi $\left(29.06441^{\circ} \mathrm{N}, 79.82281^{\circ}\right.$ E) G. B. Pant University of Agriculture and Technology, Pantnagar in the month of March.

\section{Experimental work}

\section{Preparation of plant material}

Leaves were washed thoroughly and shade dried for one week.

Then dried leaves were grounded to make powder and stored in air tight container.

\section{Preparation of samples}

Plant extracts were prepared in four different solvents viz., chloroform, ethyl acetate, acetone and methanol. Powdered plant material was used for respective solvent (1:4 w:v) extraction. The extracts were filtered, evaporated and dried then respective extracts were used for further experimentation.

\section{Analysis of total phenol content}

The Folin-Ciocalteu Method (McDonald et al., 2001) with minor modifications was used to determine the total phenolic contents of extracts using catechol as standard. The results were then expressed as $\mathrm{g}$ of catechol per $100 \mathrm{~g}$ of sample in dried weight (DW).

\section{Analysis of total flavonoid content}

The total flavanoid content was determined by the method of Mandal et al., (2009) using catechin as a standard. The total flavonoid contents were expressed as catechin equivalents in gram per $100 \mathrm{~g}$ dried sample. 
Determination of total antioxidant activity in $A$. cordifolia leaf extracts

The assay was performed according method described by Oueslati et al., 2012. The assay was based on the reduction of $\mathrm{Mo}(\mathrm{VI})$ to $\mathrm{Mo}(\mathrm{V})$ by the extract and subsequent formation of a green phosphate $\mathrm{Mo}(\mathrm{V})$ complex at acid $\mathrm{pH}$. The total antioxidant activity was expressed as the number of equivalence of ascorbic acid.

\section{Statistical analysis}

All the analyses were performed in triplicates and results were reported as means \pm standard deviation (SD). The significance of differences among treatment means were determined by one way analysis of variance (ANOVA) with a significant level of $(\mathrm{p}<$ 0.05 ). One-way analysis of variance (ANOVA) was carried out using SPSS 16.

Gas Chromatography based mass spectroscopic analysis of $A$. cordifolia extracts

\section{Interpretation of mass spectrum}

The GC-MS analysis was carried out by using GCMS-QP2010 Plus with following experimental conditions: Initial temperature $60{ }^{\circ} \mathrm{C}$ with RAMP of $3{ }^{\circ} \mathrm{C} / \mathrm{min}$, final temperature $210^{\circ} \mathrm{C}$, final hold time $10 \mathrm{~min}$, carrier gas $\mathrm{He}$, flow rate $1 \mathrm{~mL} / \mathrm{min}$. Column, silica DB-5, capillary (30nm X $0.25 \mathrm{~mm}$ ID X $0.25 \mu \mathrm{mdf})$. MS were recorded under electron ionization (EI) condition $(70 \mathrm{eV})$ with split mode of 40:1. The compounds were identified by matching their mass spectra with those recorded in NISTMS Wiley Library.

\section{Results and Discussion}

The Phenolic compounds contribute to the antioxidant potential of plants by neutralizing free radicals and preventing decomposition of hydroperoxides into free radicals. Hence, it is important to quantify phenolic derivatives and to assess its contribution to antioxidant activity. Plant extracts were prepared in four different solvents viz., chloroform, ethyl acetate, acetone and methanol having polarity index 2.7, 4.4, 5.1 and 5.1 respectively. Total Phenolic content was expressed as g catechol equivalents $/ 100 \mathrm{~g}$ dry weight. The total phenolic contents of Adina cordifolia extracts ranged from 1.86 to $8.47 \mathrm{~g} / 100 \mathrm{~g}$ in chloroform and acetone extracts respectively (Table 1). Highest total phenolic content was found in acetonic extract of leaf. The content is significantly higher than the phenolic contents of chloroform and ethyl acetate solvents used. Significant differences were recorded in the total phenolic contents of chloroform and ethyl acetate except methanol. Phenol was highest in acetone and lowest in chloroform due to polarity index of solvents. Kumari et al., (2017) reported the total phenolic content in Adina cordifolia leaves (determined as gallic acid equivalents or GAE) in ethyl acetate fraction $29.82 \pm 2.51 \mathrm{mg} \mathrm{GAE} / \mathrm{g}$ and in methanolic extract $45.32 \pm 2.67 \mathrm{mg} \mathrm{GAE} / \mathrm{g}$.

Total flavonoid content was expressed as $\mathrm{g}$ catechin equivalents/100g dry weight. The total flavonoid content of Adina cordifolia was recorded ranging from $2.21 \mathrm{~g} / 100 \mathrm{~g}$ to $9.23 \mathrm{~g} / 100 \mathrm{~g}$ in chloroform and acetone extracts respectively. The acetone extract exhibited a total flavonoid content that is significantly higher than chloroform and methanol extracts. The flavonoid contents of the rest of the solvents are also significantly different from each other except for ethyl acetate extract whose flavonoid contents are not significantly different. Flavonoid was highest in methanol and lowest in chloroform due to polarity index of solvents. Kumari et al., (2017) reported total flavonoids content in methanolic extract (39.94 $\pm 3.02 \mathrm{mg} \mathrm{Rutin} / \mathrm{g})$. The difference in amounts of phenols and flavonoids are 
probably related to geographical and environmental factors, processing methods which may play role in such a large variation.

Total antioxidant potential expressed as ascorbic acid equivalents (AAE) $\mathrm{g} / 100 \mathrm{~g}$ and in methanol extract of Adina cordifolia leaf had highest total anti-oxidant activity i.e. 36.08 $\pm 0.33 \mathrm{~g} / 100 \mathrm{~g}$ DW (Table 1). The total antioxidant potential of Adina cordifolia were recorded ranging from $23.28 \mathrm{~g} / 100 \mathrm{~g}$ to 36.08 $\mathrm{g} / 100 \mathrm{gm}$. Significant differences were recorded in all the extracts.

The dependency of antioxidant activity obtained through assay, in relationship to the Total Phenolic Content, was also evaluated. Although, there is a positive linear correlation $(\mathrm{r}=0.9221)$ among the total antioxidant activity and TPC assay for $\mathrm{R}^{2}=0.8504$. The results indicated that the phenolic compounds in the different extracts of leaf could be the main contributor to the antioxidant activities.

\section{Identification of phytocompounds by GC- MS}

Identification of phytocompounds was based on the principles of molecular weight (MW), retention time (RT), molecular formula (MF) and concentration (peak area\%).

It was done in order to determine some compounds present in plants having any medicinal value. The Gas chromatography mass spectrum of the sample were interpreted using the database of National Institute Standard and Technology (NIST) having more than 2,00,000 patterns. For identification of any unknown compound, its spectrum is compared to spectrums database stored in NIST-11 library for similarity.

A total of 66 constituents, contributing $61.74 \%$ of the chloroform extract, $80.42 \%$ of the ethyl acetate extract, $60.88 \%$ of the acetonic extract and $45.59 \%$ of the methanolic extract were identified. Persual of Table 2 indicates the names and respective percentage of identified constituents while figure 1 represents the major compounds.

All the extracts were different in their qualitative and quantitative make-up of major and minor constituents.

In present communication the dominating constituents in the leaves extracts of Haldu were trans squalene (15.4-42.1\%), vitamin $\mathrm{E}$ (2.9-5.8\%), phytol (1.1-9.42\%), and neophytadiene $(2.0-2.4 \%)$ in respective extracts.

Trans squalene and vitamin $\mathrm{E}$ possess antioxidant power.

The compounds identified in leaf $A$. cordifolia chloroform extracts are tetradecanal $(0.93 \%)$, Neophytadiene $(2.46 \%)$, Trans-squalene (42.13\%), Phytol isomer (2\%), Vitamin E (4.22\%), Ergost-5-en-3-ol (3.38\%), Campesterol (1.71\%), Naphthalene (3.48\%). Trans-squalene (15.42 \%), Neophytadiene $(2.05 \%)$, Hexadecanoic acid methyl ester $(1.10 \%)$, Phytol isomer $(9.42 \%)$, Tetradecanal (1.09\%), Vitamin E (5.84\%), Gamma.sitosterol (4.11\%) were reported in $A$. cordifolia's ethyl acetate extract.

Trans-squalene (27.44\%), Tetradecanal (0.68\%), Neophytadiene (2.09\%), Trimethylsilyl palmitate $(3.36 \%)$, Phytol isomer (1.18\%), Vitamin E (2.99\%), Campesterol (1.05\%), Phenol (7.33\%), Naphthalene $(3.77 \%)$ were reported in $A$. cordifolia's acetonic extract whereas phenol (1.14\%), naphthalene (1.16\%), Epiglobulol $(3.23 \%)$, caryophyllenoxide $(4.14 \%)$, loliolide $(1.32 \%)$, pentyl octanoate $(3.44 \%)$, behenyl behenate $(6.53 \%)$ were identified in leaf $A$. cordifolia methanolic extract. 
Table.1 Total phenolic content, total flavonoid content and total antioxidant activity in leaves of Adina cordifolia in different solvent extracts

\begin{tabular}{|c|c|c|c|}
\hline Leaf Extracts & $\begin{array}{c}\text { Total Phenolic } \\
\text { content }(\mathbf{g} \text { CalE/ } \\
\mathbf{1 0 0 g d w})\end{array}$ & $\begin{array}{c}\text { Total flavonoid } \\
\text { content }(\mathbf{g ~ C E} / \\
\mathbf{1 0 0 g d w})\end{array}$ & $\begin{array}{c}\text { Total antioxidant } \\
\text { Potential }(\mathbf{g} / \mathbf{1 0 0 g m})\end{array}$ \\
\hline Chloroform & $1.86 \pm 0.43^{\mathrm{a}}$ & $2.21 \pm 0.46^{\mathrm{a}}$ & $23.28 \pm 0.63^{\mathrm{a}}$ \\
\hline Ethyl acetate & $3.51 \pm 0.33^{\mathrm{b}}$ & $8.38 \pm 0.89^{\mathrm{c}}$ & $28.92 \pm 0.74^{\mathrm{b}}$ \\
\hline Acetone & $8.47 \pm 0.61^{\mathrm{c}}$ & $9.23 \pm 0.91^{\mathrm{c}}$ & $33.22 \pm 0.32^{\mathrm{c}}$ \\
\hline Methanol & $7.54 \pm 0.39^{\mathrm{c}}$ & $4.96 \pm 0.27^{\mathrm{b}}$ & $36.08 \pm 0.33^{\mathrm{d}}$ \\
\hline
\end{tabular}

$\mathrm{CalE}=\mathrm{Catechol}$ equivalent $\mathrm{CE}=$ Catechin equivalent Each value is expressed as mean \pm S.D (Standard Deviation) $(\mathrm{n}=3)$.

Table.2 Some phytocompound and their important uses

\begin{tabular}{|c|c|c|c|}
\hline S. N. & Name of compound & Activity & References \\
\hline 1. & $\begin{array}{l}\text { 3,5-di-tert- } \\
\text { butylphenol }\end{array}$ & Antifungal activity & Rathna, et al., 2016 \\
\hline 2. & $\begin{array}{l}\text { Trans-chrysanthemic } \\
\text { acid }\end{array}$ & $\begin{array}{l}\text { Important component of pyrethrins (Natural } \\
\text { pesticide) }\end{array}$ & Xu H. et al., 2018 \\
\hline 3. & Loliolide & $\begin{array}{l}\text { Astringent, antipyretic, anti-inflammatory and } \\
\text { vasodilatory effects }\end{array}$ & Fujita et al., 1972 \\
\hline 4. & Neophytadiene & $\begin{array}{l}\text { Antipyretic, Analgesic, And Anti-Inflammatory, } \\
\text { Antimicrobial }\end{array}$ & $\begin{array}{l}\text { Duke and Beckstrom- } \\
\text { Sternberg (1994) }\end{array}$ \\
\hline 5. & Linolenic acid & Important in prevention of coronary heart disease & Lorgeril et al., 2001 \\
\hline 6. & N-hexadecanoic acid & Anti-inflammatory property & Aparna et al., 2012 \\
\hline 7. & Vitamin E & $\begin{array}{lrr}\text { Antiageing, } & \text { Analgesic, } & \text { Antidiabatic } \\
\text { Antiinflammatory, } & \text { Antioxidant }\end{array}$ & $\begin{array}{l}\text { Duke and Beckstrom- } \\
\text { Sternberg (1994) }\end{array}$ \\
\hline 8. & Trans-squalene & $\begin{array}{l}\text { Neutralize xenobiotics, anti-inflammatory, anti- } \\
\text { atherosclerotic and anti-neoplastic, role in skin } \\
\text { aging and Adjuvant activities }\end{array}$ & $\begin{array}{l}\text { Duke and Beckstrom- } \\
\text { Sternberg (1994) }\end{array}$ \\
\hline 9. & Ergost-5-en-3-ol & $\begin{array}{l}\text { Anti-inflammatory, Analgesic and Antipyretic } \\
\text { property }\end{array}$ & Kanthel et al., 2014 \\
\hline 10. & Campesterol tms & $\begin{array}{l}\text { Antimicrobial Anti-inflammatory Anticancer } \\
\text { Antiarthritic Antiasthma Diuretic }\end{array}$ & $\begin{array}{l}\text { Duke and Beckstrom- } \\
\text { Sternberg (1994) }\end{array}$ \\
\hline 11. & Gamma-sitosterol & $\begin{array}{l}\text { Anti-diabetic, Anti-angeogenic, anticancer, } \\
\text { antimicrobial, anti-inflammatory, antidiarrhoeal and } \\
\text { antiviral }\end{array}$ & $\begin{array}{l}\text { Duke and Beckstrom- } \\
\text { Sternberg (1994)) }\end{array}$ \\
\hline 12. & $\begin{array}{l}\text { 9-octadecenoic acid, } \\
\text { methyl ester }\end{array}$ & $\begin{array}{l}\text { Antiinflammatory, Hypocholesterolemic Cancer } \\
\text { preventive, Hepatoprotective, Nematicide } \\
\text { Insectifuge, Antihistaminic Antieczemic, Antiacne, } \\
\text { 5-Alpha reductase inhibitor Antiandrogenic, } \\
\text { Antiarthritic, Anticoronary, Insectifuge }\end{array}$ & $\begin{array}{l}\text { Duke and Beckstrom- } \\
\text { Sternberg (1994) }\end{array}$ \\
\hline 13. & $\begin{array}{l}\text { Beta-sitosterol } \\
\text { trimethyl silyl ether }\end{array}$ & $\begin{array}{l}\text { Antimicrobial Anti-inflammatory Anticancer } \\
\text { Antiarthritic Antiasthma Diuretic }\end{array}$ & $\begin{array}{l}\text { Duke and Beckstrom- } \\
\text { Sternberg (1994) }\end{array}$ \\
\hline
\end{tabular}


Fig.1 Adina cordifolia's leaf total phenolic and flavonoid content in different solvent extracts

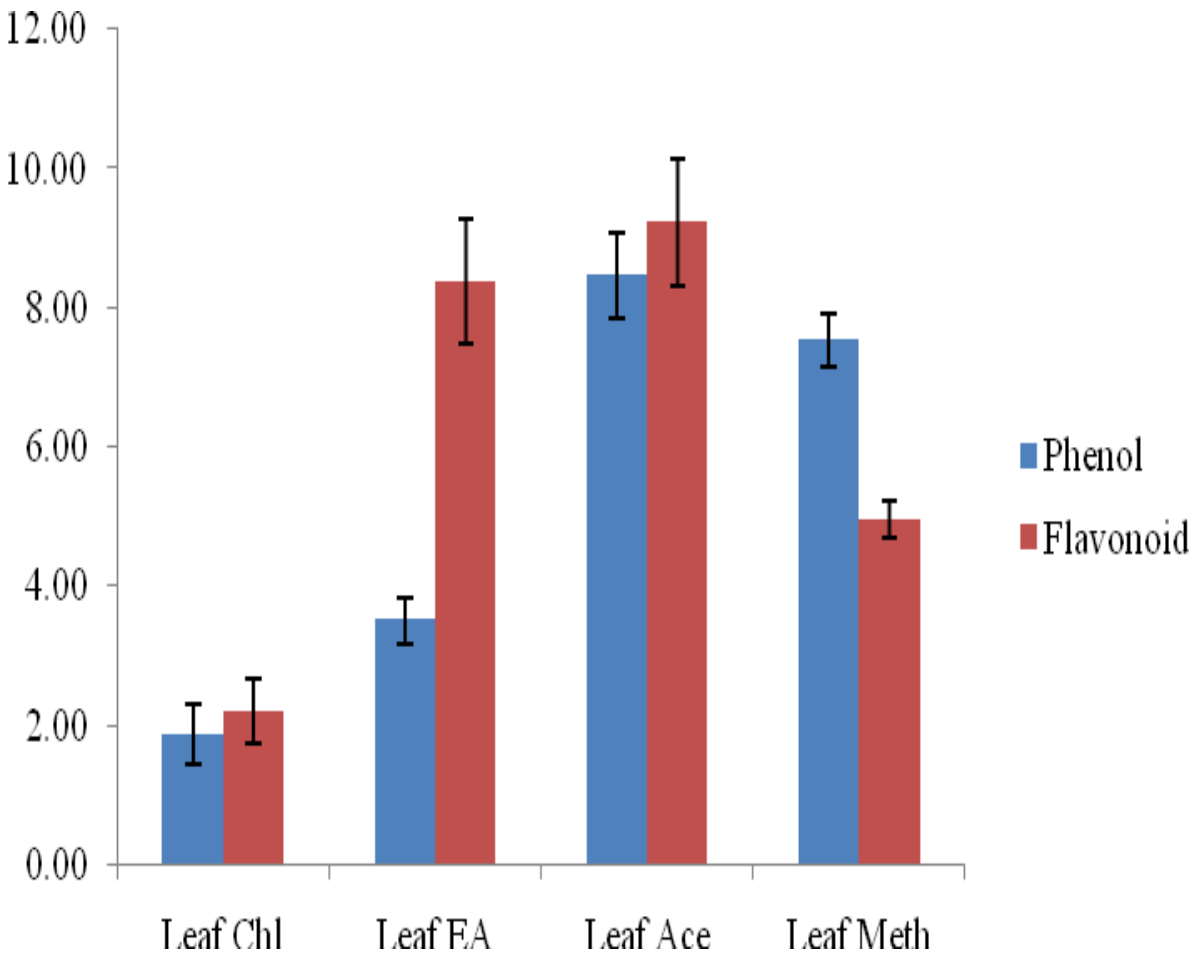

Fig.2 Adina cordifolia's leaf total total antioxidant activity in different solvent extracts

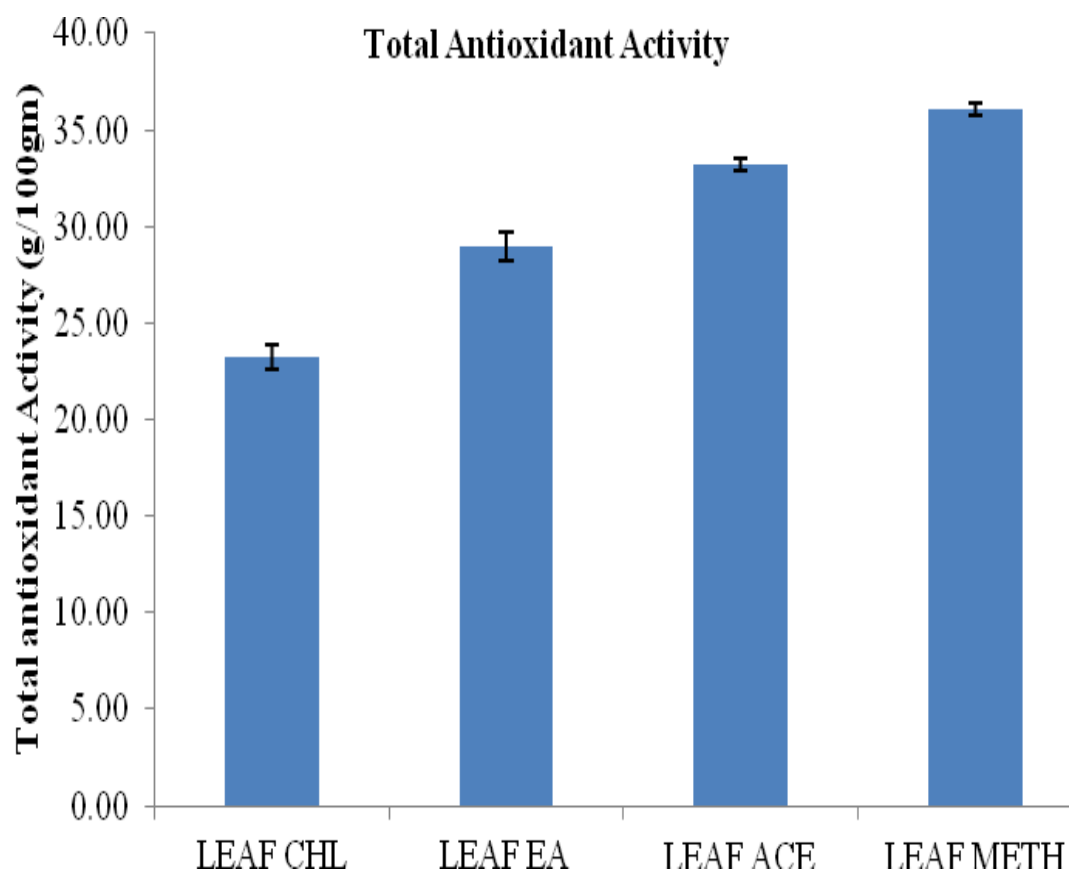


Fig.3 GC MS analysis chromatogram for Adina cordifolia's leaf Chloroform extract

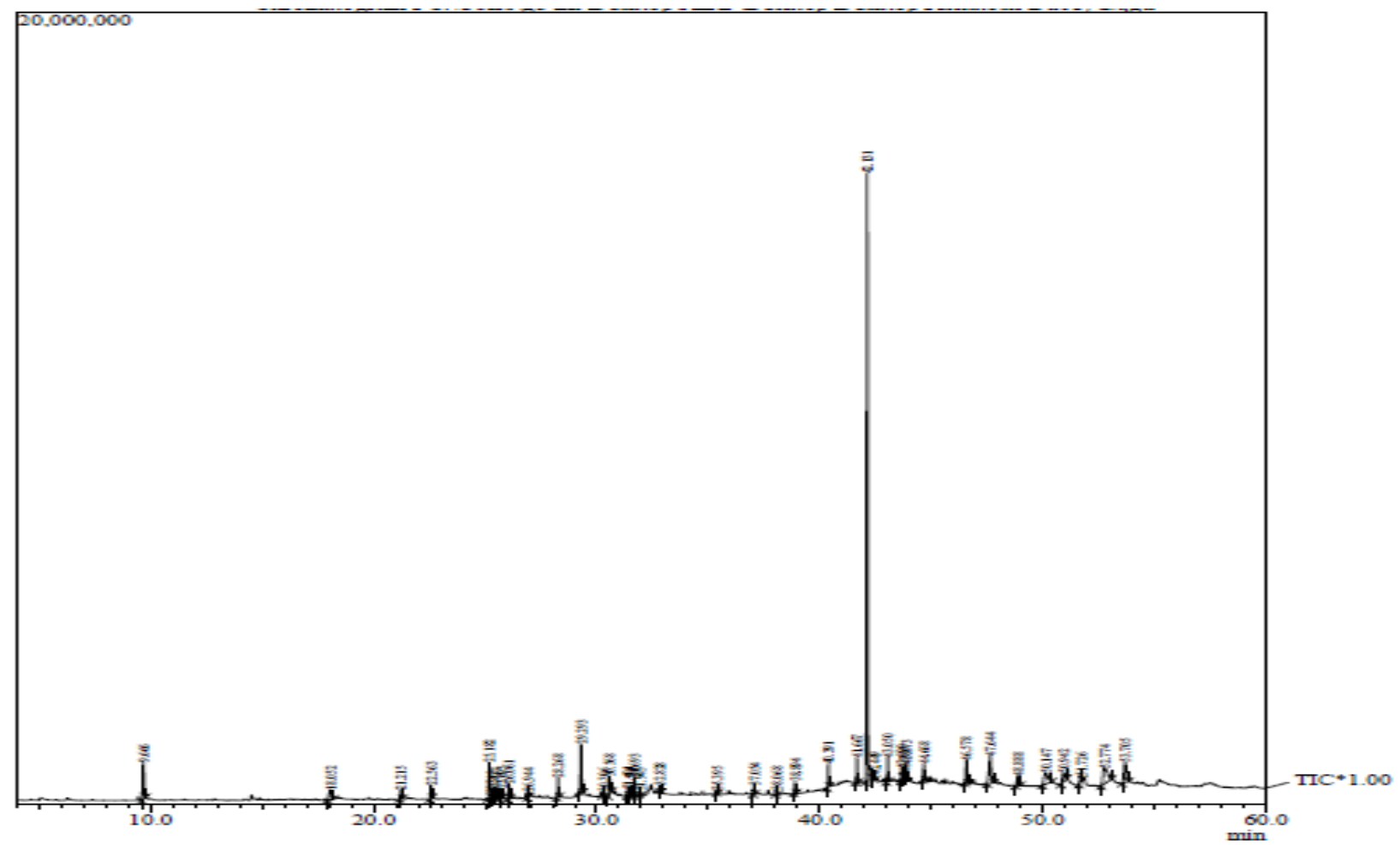

Fig.4 GC MS analysis chromatogram for Adina cordifolia's leaf Ethyl acetate extract

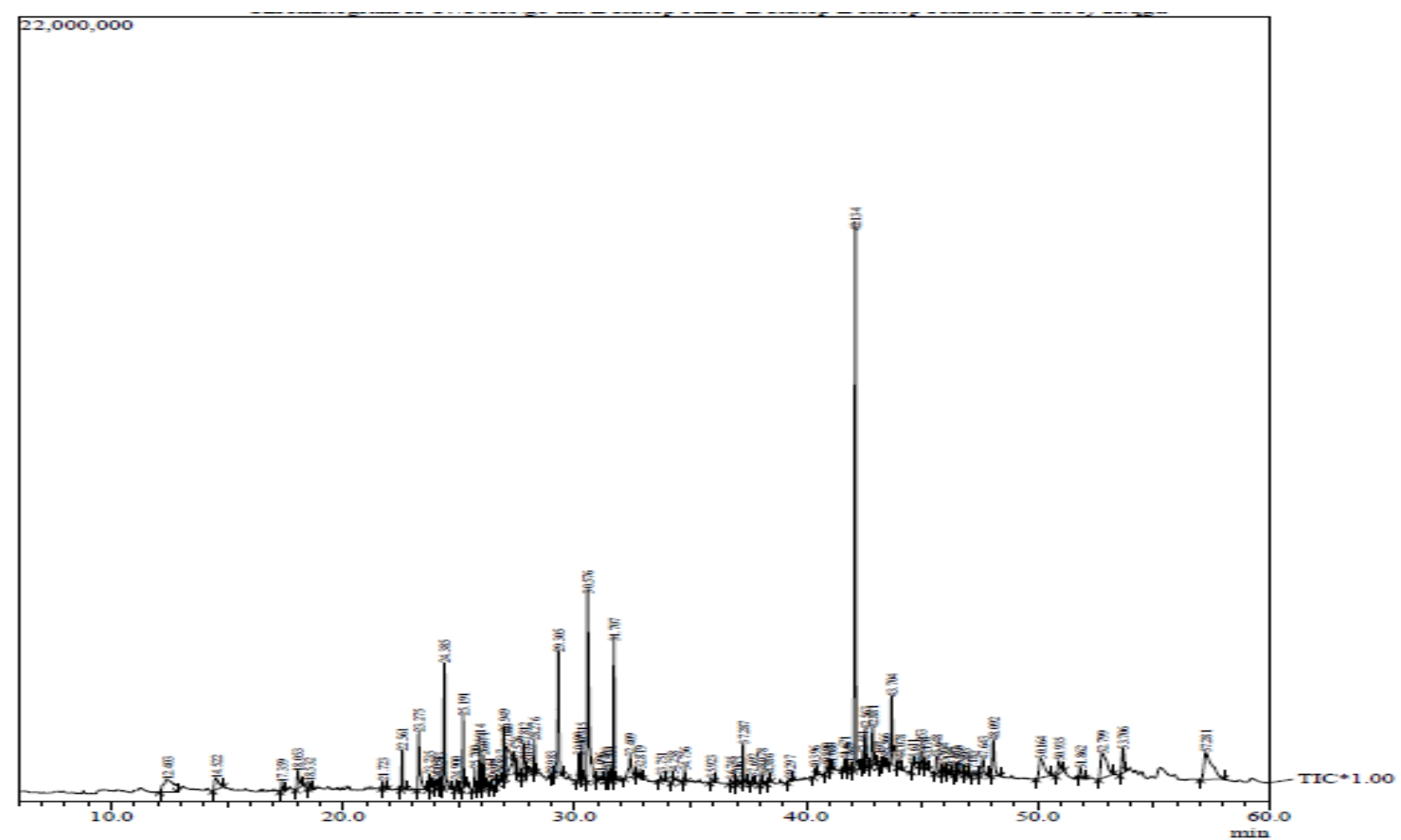


Fig.5 GC-MS analysis chromatogram for Adina cordifolia's leaf Acetonic extract

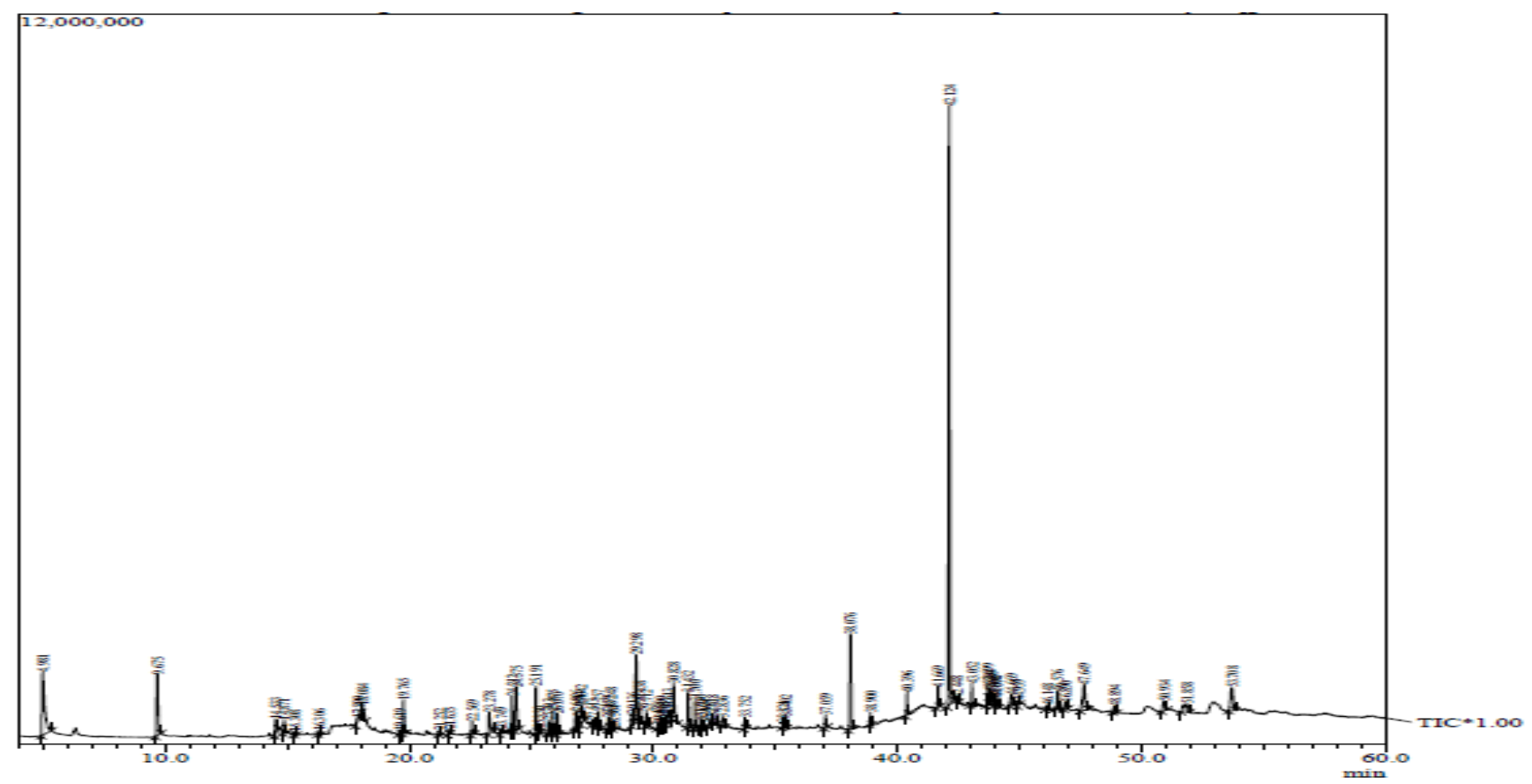

Fig.6 GC-MS analysis chromatogram for Adina cordifolia's leaf methanolic extract

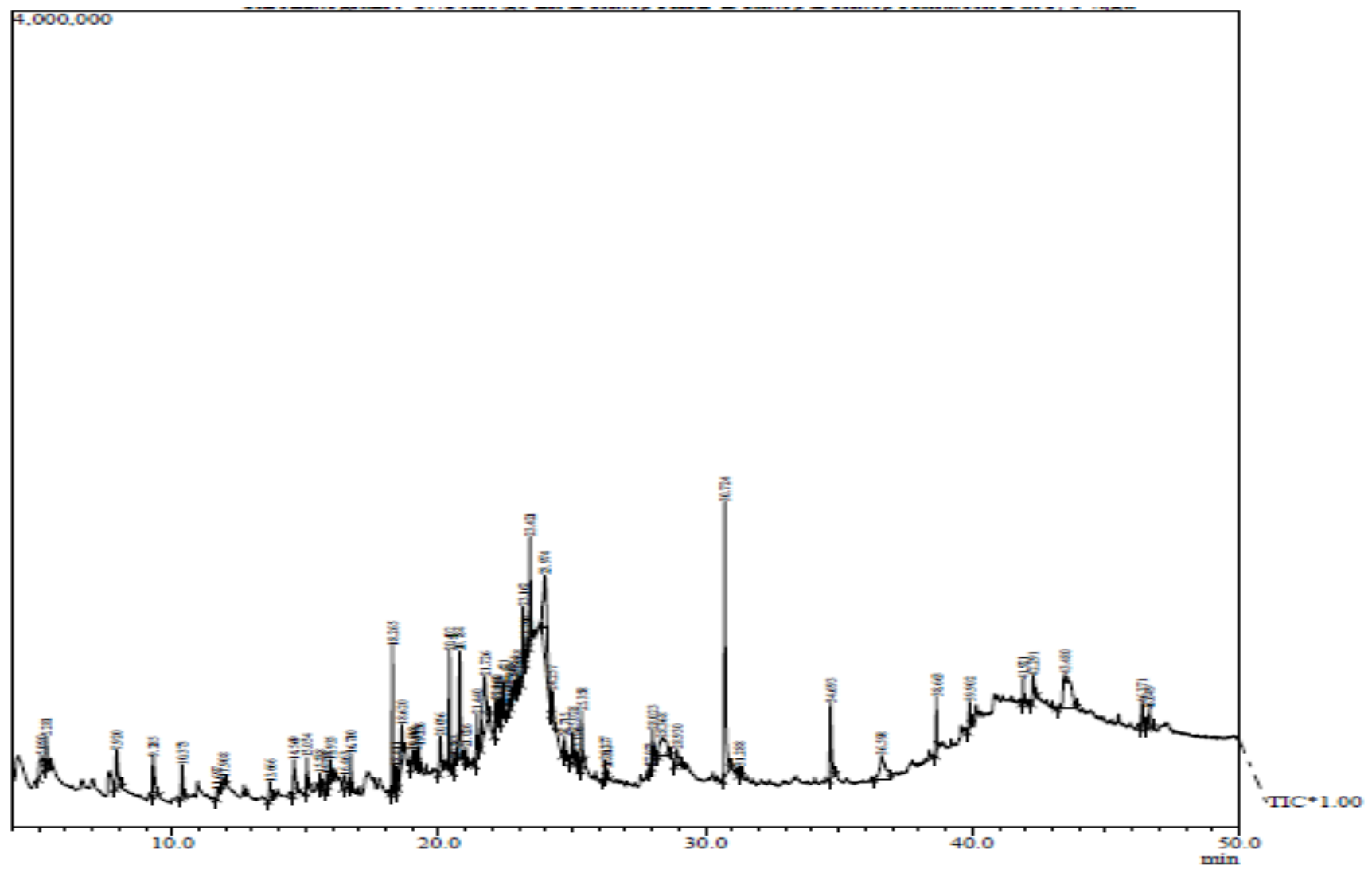


Comparative analysis of Adina cordifolia's Leaves Metabolites

\begin{tabular}{|c|c|c|c|c|c|c|}
\hline \multicolumn{7}{|c|}{ Comparative analysis of Adina cordifolia's Leaves Metabolites } \\
\hline Compound & $\begin{array}{l}\text { Molecular } \\
\text { Formula }\end{array}$ & $\begin{array}{l}\text { Nature of } \\
\text { Compound }\end{array}$ & $\begin{array}{c}\text { \% Contribution } \\
\text { in Chloroform } \\
\text { Extract }\end{array}$ & $\begin{array}{c}\% \text { Contribution in } \\
\text { Ethyl actate } \\
\text { extract }\end{array}$ & $\begin{array}{c}\% \text { Contribution } \\
\text { in Acetonic } \\
\text { extract }\end{array}$ & $\begin{array}{c}\% \\
\text { Contribution in } \\
\text { Methanol } \\
\text { extract }\end{array}$ \\
\hline Methyl 4-ethylbenzoate & $\mathrm{C}_{10} \mathrm{H}_{12} \mathrm{O}_{2}$ & Monoterpenoid & & 1.33 & & \\
\hline 3,5-di-tert-butylphenol & $\mathrm{C}_{14} \mathrm{H}_{22} \mathrm{O}$ & Phenol & & 1.09 & 0.82 & \\
\hline Trans-chrysanthemic acid & $\mathrm{C}_{10} \mathrm{H}_{16} \mathrm{O}_{2}$ & Monoterpenoid & & 0.33 & & \\
\hline Tetradecanal & $\mathrm{C}_{14} \mathrm{H}_{28} \mathrm{O}$ & Aldehyde & 0.93 & 1.09 & 0.68 & \\
\hline P-menth-3-en-9-ol & $\mathrm{C}_{10} \mathrm{H}_{18} \mathrm{O}$ & Monoterpenoid & & 0.49 & & \\
\hline Loliolide & $\mathrm{C}_{11} \mathrm{H}_{16} \mathrm{O}_{3}$ & Carotenoid & & 0.34 & & 1.32 \\
\hline $\begin{array}{l}\text { 2,5,5,8a-tetramethyl-6,7,8,8a-tetrahydro-5h- } \\
\text { chromen-3-one }\end{array}$ & $\mathrm{C}_{13} \mathrm{H}_{20} \mathrm{O}_{2}$ & Ketone & & 4.51 & & \\
\hline Neophytadiene & $\mathrm{C}_{20} \mathrm{H}_{38}$ & Diterpenoid & 2.46 & 2.05 & 2.09 & \\
\hline 2-methyl-octadecyne & $\mathrm{C}_{19} \mathrm{H}_{36}$ & Hydrocarbon & & 0.66 & & \\
\hline Dodecanal dimethylacetal & $\mathrm{C}_{14} \mathrm{H}_{30} \mathrm{O}_{2}$ & Aldehyde & & 1.10 & & \\
\hline 3,7,11,15-tetramethyl-2-hexadecen-1-ol & $\mathrm{C}_{20} \mathrm{H}_{40} \mathrm{O}$ & Diterpenoid & & 1.66 & & \\
\hline Citronellol acetate & $\mathrm{C}_{12} \mathrm{H}_{22} \mathrm{O}_{2}$ & Hydrocarbon & & 0.39 & & \\
\hline Hexadecanoic acid methyl ester & $\mathrm{C}_{17} \mathrm{H}_{34} \mathrm{O}_{2}$ & Ester & 0.32 & 1.10 & & \\
\hline Dimethyl $\{$ bis $[(2 z)$-pent-2-en-1-yloxy $]\}$ silane & $\mathrm{C}_{12} \mathrm{H}_{24} \mathrm{O}_{2} \mathrm{Si}$ & Hydrocarbon & & 2.96 & & \\
\hline N-hexadecanoic acid & $\mathrm{C}_{16} \mathrm{H}_{32} \mathrm{O}_{2}$ & Fatty acid & & 1.38 & & \\
\hline 2-hydroxyisocaproic acid, trimethylsilyl ester & $\mathrm{C}_{9} \mathrm{H}_{20} \mathrm{O}_{3} \mathrm{Si}$ & Ester & & 0.37 & & \\
\hline Ethyl pentadecanoate & $\mathrm{C}_{17} \mathrm{H}_{34} \mathrm{O}_{2}$ & Hydrocarbon & & 0.74 & & \\
\hline Trimethylsilyl palmitate & $\mathrm{C}_{19} \mathrm{H}_{40} \mathrm{O}_{2} \mathrm{Si}$ & Hydrocarbon & & 4.60 & 3.36 & \\
\hline Linoleic acid, methyl ester & $\mathrm{C}_{19} \mathrm{H}_{34} \mathrm{O}_{2}$ & Ester & & 0.65 & & \\
\hline Phytol isomer & $\mathrm{C}_{20} \mathrm{H}_{40} \mathrm{O}$ & Diterpenoid & 2.00 & 9.42 & 1.18 & \\
\hline N-propyl 9,12-octadecadienoate & $\mathrm{C}_{21} \mathrm{H}_{38} \mathrm{O}_{2}$ & Hydrocarbon & & 0.28 & & \\
\hline Phytol, trimethylsilyl ether & $\mathrm{C}_{23} \mathrm{H}_{48} \mathrm{OSi}$ & Hydrocarbon & 1.11 & 3.35 & & \\
\hline N-octadecyl isocyanate & $\mathrm{C}_{19} \mathrm{H}_{37} \mathrm{NO}$ & Hydrocarbon & & 1.35 & & \\
\hline Hexadecanal & $\mathrm{C}_{16} \mathrm{H}_{32} \mathrm{O}$ & Aldehyde & & 0.27 & & \\
\hline Isooctyl phthalate & $\mathrm{C}_{24} \mathrm{H}_{38} \mathrm{O}_{4}$ & Hydrocarbon & & 0.40 & & \\
\hline Tetradecanal & $\mathrm{C}_{14} \mathrm{H}_{28} \mathrm{O}$ & Aldehyde & & 0.25 & & \\
\hline Solanesol & $\mathrm{C}_{45} \mathrm{H}_{74} \mathrm{O}$ & Alcohols & & 1.75 & & \\
\hline Geranyl linalool isomer & $\mathrm{C}_{20} \mathrm{H}_{34} \mathrm{O}$ & Diterpenoid & & 0.56 & & \\
\hline
\end{tabular}




\begin{tabular}{|c|c|c|c|c|c|c|}
\hline Neryl linalool isomer & $\mathrm{C}_{20} \mathrm{H}_{34} \mathrm{O}$ & Diterpenoid & & 0.38 & & \\
\hline Beta.-tocopherol & $\mathrm{C}_{28} \mathrm{H}_{48} \mathrm{O}_{2}$ & Phenols & & 0.80 & & \\
\hline Gamma.-tocopherol & $\mathrm{C}_{28} \mathrm{H}_{48} \mathrm{O}_{2}$ & Phenols & & 0.36 & & \\
\hline 1-bromotetracosane & $\mathrm{C}_{24} \mathrm{H}_{49} \mathrm{Br}$ & Hydrocarbon & & 0.23 & & \\
\hline Methoprene & $\mathrm{C}_{19} \mathrm{H}_{34} \mathrm{O}_{3}$ & Hydrocarbon & & 2.25 & & \\
\hline Ergost-5-en-3-ol & $\mathrm{C}_{28} \mathrm{H}_{48} \mathrm{O}$ & Sterols & 3.38 & 2.79 & & \\
\hline Campesterol tms & $\mathrm{C}_{31} \mathrm{H}_{56} \mathrm{OSi}$ & Sterols & 1.71 & 0.99 & 1.05 & \\
\hline Beta.-sitosterol trimethylsilyl ether & $\mathrm{C}_{32} \mathrm{H}_{58} \mathrm{OSi}$ & Sterols & & 1.58 & & \\
\hline Phenol & $\mathrm{C}_{6} \mathrm{H}_{6} \mathrm{O}$ & Phenol & & & 7.33 & 1.14 \\
\hline Naphthalene & $\mathrm{C}_{10} \mathrm{H}_{8}$ & Monoterpenoid & 3.48 & & 3.77 & 1.16 \\
\hline 4-trimethylsiloxybenzaldehyde & $\mathrm{C}_{10} \mathrm{H}_{14} \mathrm{O}_{2} \mathrm{Si}$ & Monoterpenoid & & & 1.02 & \\
\hline 9-octadecene & $\mathrm{C}_{18} \mathrm{H}_{36}$ & Hydrocarbon & & & 0.57 & \\
\hline 9-eicosene & $\mathrm{C}_{20} \mathrm{H}_{40}$ & Diterpenoid & & & 1.44 & \\
\hline Dodecylcyclohexane & $\mathrm{C}_{18} \mathrm{H}_{36}$ & Hydrocarbon & & & 0.20 & \\
\hline Palmitic acid, methyl ester & $\mathrm{C}_{17} \mathrm{H}_{34} \mathrm{O}_{2}$ & Fatty acid & & & 0.39 & \\
\hline $\begin{array}{l}\text { Alpha.-d-xylofuranose, 1,2-0-isopropylidene-5-(t- } \\
\text { butyldimethylsilyl)- }\end{array}$ & $\mathrm{C}_{14} \mathrm{H}_{28} \mathrm{O}_{5} \mathrm{Si}$ & Carbohydrate & & & 1.53 & \\
\hline 1-heneicosanol & $\mathrm{C}_{21} \mathrm{H}_{44} \mathrm{O}$ & Alcohols & & & 0.74 & \\
\hline Tetratriacontane & $\mathrm{C}_{34} \mathrm{H}_{70}$ & Hydrocarbon & & & 0.13 & \\
\hline Trimethylsilyl 3-phenoxypropanoate & $\mathrm{C}_{12} \mathrm{H}_{18} \mathrm{O}_{3} \mathrm{Si}$ & Ester & & & 1.01 & \\
\hline 2,4'-bis(trimethylsilyloxy)diphenylmethane & $\mathrm{C}_{19} \mathrm{H}_{28} \mathrm{O}_{2} \mathrm{Si}$ & Hydrocarbon & & & 0.66 & \\
\hline Ethyl 9,12-hexadecadienoate & $\mathrm{C}_{18} \mathrm{H}_{32} \mathrm{O}_{2}$ & Ester & & 0.23 & & \\
\hline 9-octadecenoic acid, methyl ester & $\mathrm{C}_{19} \mathrm{H}_{36} \mathrm{O}_{2}$ & Ester & & 0.33 & & \\
\hline Methyl ricinoleate & $\mathrm{C}_{19} \mathrm{H}_{36} \mathrm{O}_{3}$ & Fatty acid & & 0.17 & & 3.12 \\
\hline Behenyl behenate & $\mathrm{C}_{44} \mathrm{H}_{88} \mathrm{O}_{2}$ & Ester & & & & 6.53 \\
\hline mome inositol & $\mathrm{C}_{7} \mathrm{H}_{14} \mathrm{O}_{6}$ & Alcohol & & & & 8.18 \\
\hline Pentyl octanoate & $\mathrm{C}_{13} \mathrm{H}_{26} \mathrm{O}_{2}$ & Ester & & & & 3.44 \\
\hline 13-Hexyloxacyclotridec-10-en-2-one & $\mathrm{C}_{18} \mathrm{H}_{32} \mathrm{O}_{2}$ & Ketone & & & & 9.81 \\
\hline Pentyl octanoate & $\mathrm{C}_{13} \mathrm{H}_{26} \mathrm{O}_{2}$ & Ester & & & & 3.44 \\
\hline
\end{tabular}


The compounds 3,5 ditert-butylphenol, transchrysanthemic acid, loliolide, neophytadine, Hexadecanoic acid, Vitamin E, Transsqualene, Ergost-5-en-3-ol, Campesterol, Gamma.-sitosterol, 9- octadecanoic acid methyl ester, beta sitosterol trimethyl silyl ether etc. identified in present study have been reported to used as starting material for the synthesis of industrially important component of natural pesticide (Xu et al., 2018), possess antifungal activity, astringent power, antipyretic property, inflammatory effect, analgesic effect, vasodilatory effect, antimicrobial property, antiageing and antidiabetic property, antioxidant activity, adjuvant activities, antiarthritic, antidiarrhoel and antiviral effect (Rathna et al., 2016; Fujita et al., 1972; Lorgeril et al., 2001; Aparna et al., 2012; Kanthal et al., 2014; Duke and Beckstrom-Sternberg, 1994).

Based on these findings it can be concluded that the plant may be good natural source for many industrially important phytoconstituents. The plant can also be a source of herbal medicine and natural antioxidant.

The results of present study indicated considerable amount of total phenolic content and total flavonoid content. The highest total phenolic and flavonoid content were recorded in leaf acetone extract. According to Verma et al., (2010) flavonoids and alkaloids seem to be most likely compounds eliciting in vitro cytotoxicity effect. The phenolic compounds are reported to show as scavengers of Reactive Oxygen Species (ROS), antioxidant and anti-inflammatory activities (Sivanandham, 2011). The flavonoids are also medicinally important and exhibit analgesic, anti-inflammatory, antioxidant, anti-arthritic and immunomodulatory properties (Gill et al., 2011). Total antioxidant potential was found highest in methanol extract of leaf $A$. cordifolia due to some compounds having antioxidant property such as loliolide, methyl ricinoleate.

In this study different extracts of Adina cordifolia leaves were analyzed for the presence of active bioactive compounds by GC-MS analysis with their spectrum, retention time, molecular weight and similarity index. The mass spectrum of each compound was compared with NIST-11 database and gave more than $90 \%$ match resulting in confirmatory compound match. Major component found in the extracts is squalene, isoprenoid compound that possesses antioxidant activities and widely produced in plants. Squalene protects cells against radicals, strengthens the immune system and decreases the risk of various cancers (Kalinova et al., 2006). Squalene is not very susceptible to peroxidation and appears to function in the skin as a quencher of singlet oxygen, protecting human skin surface from lipid peroxidation due to exposure to UV and other sources of ionizing radiation.

Chloroform is best solvent for extraction of squalene followed by acetone and ethyl acetate while methanol is not capable of extracting squalene. Table 2 contains important phytocompounds found in Adina cordifolia leaf and their biological activity. The activity of compound was identified from Dr. Duke's Phytochemical and Ethnobotanical database (Duke and Beckstrom-Sternberg, 1994).

Leaf juice of Adina cordifolia is used to treat boils and eye disorders like conjunctivitis (Hossan 2009). There are no reports available on the identification of biologically important compounds from Adina cordifolia. In this study, chloroform, ethyl acetate, acetonic and methanolic extract of Adina cordifolia leaf were quantitatively analyzed for total phenolic and flavonoid content and total antioxidant activity and then active bioactive 
compounds of plants were evaluated by GCMS analysis. The important compounds identified by GC-MS belong to diterpene, triterpene and fatty acids. These identified phytocompounds are assumed to be responsible for eliciting the traditional medicinal activity of $A$. cordifolia. The present study is significant because there is less literature available on Adina cordifolia phytocompound analysis.

\section{Acknowledgement}

Authors are grateful to Dept. of Biochemistry, GBPUA\&T, Pantnagar to carry out this research work. Our sincere thanks to DSTFIST for providing equipment facility and Directorate of Experiment Station (DES) Pantnagar for utilization of infrastructure. Pratima acknowledge UGC for fellowship.

\section{References}

Aparna, V., Dileep, K. V., Mandal, P. K., Karthe, P., Sadasivan, C. and Haridas, M. (2012). Anti-inflammatory property of n-hexadecanoic acid: structural evidence and kinetic assessment. Chemical biology \& drug design, 80(3), 434-439.

Chanda, S. and Dave, R. 2009. In vitro models for antioxidant activity evaluation and some medicinal plants possessing antioxidant properties: An overview, African Journal of Microbiology Research, 3(13): 981-996.

Chopra, R. N., Nayar, S. L. and Chopra, I. C. 1956. Glossary of Indian medicinal plants. Planta Medica, 43: 59-63.

Duke, J. A. and Beckstrom-Sternberg, S. M. 1994. Dr. Duke's Phytochemical and Ethnobotanical Databases.

Fujita, E., Saeki, Y., Ochiart, M., Inoue, T. 1972. Investigation of the Neutral Constituents of Lythrum salicaria L. Bulletin of the Institute for Chemical Research, 50: 327-331.

Gill, N. S., Arora, R., Kumar, S. R., 2011. Evaluation of antioxidant, anti- inflammatory and analgesic potential of the Luffa acutangula Roxb. var amara. Res. J. Phytochem. 5: 201-208.

Grabarczyk, M., Winska, K., Mączka, W., Potaniec, B. and Anioł, M. 2015. Loliolide-the most ubiquitous lactone. Folia Biologica et Oecologica, 11(1): 1-8. Hossan S, Hanif A, Khan M, Bari S, Jahan R, Rahmatullah M. 2009. Ethnobotanical survey of the Tripura tribe of Bangladesh. American-Euras. Journal of Sustainable Agriculture, 3(2):253-261.

Iqbal, P.F., Bhat, A. R. and Azam, A. 2009. Antiamoebic coumarins from the root bark of Adina cordifolia and their new thiosemicarbazone derivatives. European Journal of Medicinal Chemistry, 44: 2252-2259.

Kalinova, J., Triska, J. and Vrchotova, N. 2006. Distribution of vitamin $\mathrm{E}$, squalene, epicatechin, and rutin in common buckwheat plants (Fagopyrum esculentum Moench). Journal of agricultural and food chemistry, 54(15): 5330-5335.

Kanthel L. K., Dey, A. Satyavathi, K., Bhojaraju, P. 2018. GC-MS analysis of bio-active compounds in methanolic extract of Lactuca runcinata DC. Pharmacognosy Research, 6: 58-61.

Kasinadhuni, V. R. R., Rajashekhar, G., Rajagopalan, R. Sharma, V. M., Vamsi Krishna, C., Sairam, P., Sai Prasad, G., Sadhukhan, S. and Gangadhar Rao, G. 1999. Anti-ulcer potential of Haldinia cordifolia. Fitoterapia, 70: 93-95.

Kelly, G. S. 1999. Squalene and its potential clinical uses. Altern Med Rev., 4(1):2936.

Lorgeril, M. De, Salen, P., Laporte, F. and Leiris, J. de 2001. Alpha-linolenic acid in the prevention and treatment of coronary heart disease. European Heart Journal Supplements 3: 26-32.

Mandal, P., Mishra, T. K. and Ghosal, M. 2009. Free-radical scavenging activity and phytochemical analysis in the leaf and stem of Drymaria diandra Blume. 
International Journal of Integrative Biology, 7: 80-84.

McDonald, S., Prenzler, P. D., Autolovich, M., Robards, K. 2001. Phenolic content and antioxidant activity of olive extracts. Food Chem. 73: 73-84.

Oueslati, S., Trabelsi, N., Boulaaba, M., Legault, J., Abdelly, C. and Ksouri, R. 2012. Evaluation of antioxidant activities of the edible and medicinal Suaeda species and related phenolic compounds. Ind Crop Prod. 36: 513-518.

Purushoth Prabhu, T., Panneerselvam, P., Suresh, R., Clement Atlee, W., Balasubramanian. S. 2013. GC-MS analysis of ethanolic extract of Canthium parviflorum Lamk Leaf. Journal of Applied Pharmaceutical Science, 3 (02):166-168.

Raman, B. V., Samuel, L. A., Saradhi, M. P., Rao, B. N., Krishna, N. V., Sudhakar, M., and Radhakrishnan, T. M. 2012. Antibacterial, antioxidant activity and GC-MS analysis of Eupatorium odoratum. Asian Journal of Pharmaceutical and Clinical Research, 5(2): 99-106.

Rathna, N., Bakkiyara, D. and Shunmugiah, P. 2016. Anti-biofilm mechanisms of 3, 5di-tert-butylphenol against clinically relevant fungal pathogens. Biofouling, 32(9): 979-993.

Raypa, P., Verma, A. K., Dubey, A. and Tewari, S. K. 2013. In vitro Establishment of a Threatened Plant Species Adina Cordifolia. Octa Journal of Biosciences, 1(2): 138-142.

Rokade, S. and Pawar, S. P. 2013. A Comprehensive Review on Adina cordifolia. International Journal of Pharmaceutical Sciences Review and Research, 18: 13-16.

Sabir, M. and Razdan, M. K. 1970. Antifertility study with leaf extract of Adina cordifolia (Karam ki Gaach). Indian J Physiol Pharmacol; 14: 209-210.

Sivanandham, V. 2011. Free radicals in health and diseases-a mini review. Pharmacol. Onl. 1: 1062-1077.

Vasmatkar, P., Dubey A., Tyagi, B., Baral, P., Tandon, S., Kadam, A. 2014. Antibacterial activity and GC-MS analysis of methanolic extract from stem bark and leaves of mitragyna parvifolia (roxb.) korth. Indo American Journal of Pharmaceutical Research, 4: (1) 304-311.

Verma, S. K., Singh, S. K., Mathur, A., Singh, S. 2010. In vitro cytotoxicity of Argemone mexicana against different human cancer cell lines. Int. J. Chem. Env. Pharm. Res. 1 (1): 37-39.

www.fes.org.in Agar, Madhya Pradesh. 20/07/2018.

Xu, H., Lybrand, D., Bennewitz, S. Tissier, A., Last, R. L. And Pichersky, E. 2018. Production of trans-chrysanthemic acid, the monoterpene acid moiety of natural pyrethrin insecticides, in tomato fruit. Metabolic Engineering 47: 271-278.

Yoon, M. A., Jeong, T. S., Park, D. S., Xu, M. Z., Oh, H. W., Song, K. B., Lee, W. S. and Park, H. Y. 2006. Antioxidant effects of quinoline alkaloids and 2, 4-di-tertbutylphenol isolated from Scolopendra subspinipes. Biological and Pharmaceutical Bulletin, 29(4): 735-739.

\section{How to cite this article:}

Pratima Raypa, A.K. Verma, Salil Tewari and Ashutosh Dubey. 2018. Analysis of Medicinally Important Phytocompounds from Adina cordifolia Leaves. Int.J.Curr.Microbiol.App.Sci. 7(11): 3007-3019. doi: https://doi.org/10.20546/ijcmas.2018.711.345 